La responsabilidad social empresarial vista desde un enfoque teórico

DOI: $10.23857 /$ dc.v4i1.767

Número Publicado el 15 de enero de 2018

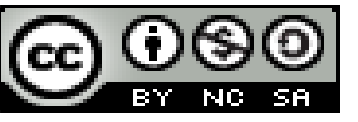

Ciencias económicas y empresariales

Artículo de investigación

\title{
La responsabilidad social empresarial vista desde un enfoque teórico
}

\section{Corporate social responsibility seen from a theoretical perspective}

\section{Responsabilidade social corporativa vista de uma perspectiva teórica}

\author{
Mónica M. Remache-Rubio ${ }^{\mathrm{I}}$ \\ mmremache2@espe.edu.ec \\ Silvia Villacis-Torres II \\ angelita.salazar@utc.edu.ec \\ Nilton A. Guayta-Toapanta ${ }^{\text {III }}$ \\ maguayta@espe.edu.ec
}

Recibido: 07 de agosto de 2017 * Corregido: 10 de octubre de $2017 *$ Aceptado: 11 de noviembre de 2017

I. Magíster en Administración de Empresas mención Planificación, Diplomado en Didáctica de la Educación Superior, Ingeniera En Organización de Empresas, Docente de la Universidad de las Fuerzas Armadas ESPE, Latacunga, Ecuador.

I. Magister en Gerencia de Proyectos Educativos y Sociales, Especialista en Liderazgo y Gerencia, Diplomado Superior en Diseño de Proyectos, Licenciada en Ciencias Administrativas Administrador de Empresas, Ingeniera de Empresas, Docente de la Universidad de las Fuerzas Armadas ESPE, Latacunga, Ecuador.

III. Ingeniero Comercial, Docente de la Universidad de las Fuerzas Armadas ESPE, Latacunga, Ecuador. 


\title{
Resumen
}

La responsabilidad social empresarial o corporativa (RSC) es la contribución voluntaria a la mejora social, económica y ambiental de las empresas, con el objetivo de mejorar su valoración competitiva y su valor añadido. Este trabajo presenta como objetivo exponer teóricamente el tema de la responsabilidad social empresarial. Para ello muestra qué se entiende por responsabilidad social, aborda el proceso histórico de la responsabilidad social; explicita los problemas actuales por acciones no responsables socialmente, los factores que afectan los sentimientos del grupo empresarial y los factores que se oponen a la productividad; se tratan los actos responsables de la empresa y las ventajas de la responsabilidad social de las empresas. Por medio del análisis documental es que se realiza este trabajo, donde se localiza bibliografía relevante, se analiza y se sintetizan los objetivos de lo general a lo particular.

Palabras clave: responsabilidad social empresarial; empresas socialmente responsables.

\begin{abstract}
Corporate or corporate social responsibility (CSR) is the voluntary contribution to the social, economic and environmental improvement of companies, with the aim of improving their competitive valuation and their added value. This work aims to theoretically expose the issue of corporate social responsibility. To do this, it shows what is understood by social responsibility, addresses the historical process of social responsibility; explains the current problems for actions not socially responsible, the factors that affect the feelings of the business group and the factors that oppose productivity; the responsible acts of the company and the advantages of the social responsibility of the companies are treated. Through documentary analysis, this work is carried out, where relevant bibliography is located, the objectives of the general to the particular are analyzed and synthesized.
\end{abstract}

Keywords: Corporate social responsibility; socially responsible companies.

\section{Resumo}

A responsabilidade social corporativa ou corporativa (CSR) é o contributo voluntário para a melhoria social, econômica e ambiental das empresas, com o objetivo de melhorar sua avaliação competitiva e seu valor agregado. Este trabalho pretende expor teoricamente a questão da responsabilidade social corporativa. Para fazer isso, mostra o que é entendido pela responsabilidade social, aborda o processo histórico de responsabilidade social; explica os problemas atuais de ações não socialmente 
La responsabilidad social empresarial vista desde un enfoque teórico

responsáveis, os fatores que afetam os sentimentos do grupo empresarial e os fatores que se opõem à produtividade; são tratados os atos responsáveis da empresa e as vantagens da responsabilidade social das empresas. Através da análise documental, este trabalho é realizado, onde a bibliografia relevante está localizada, os objetivos do geral para o particular são analisados e sintetizados.

Palavras chave: Responsabilidade social corporativa; empresas socialmente responsáveis.

\section{Introducción}

La Responsabilidad Social Empresarial se puede definir como "la capacidad de valorar las consecuencias que tienen en la sociedad las acciones y decisiones que toman las diferentes personas y organizaciones como parte del logro de sus propios objetivos y metas". (García, 2014)

Las organizaciones deben identificarse con lo que representa la responsabilidad social, con el rol de la empresa en su participación activa en el entorno en donde se desenvuelve, de acuerdo a su misión y compromiso social, aquí se incluyen también las Pymes.

En la medida que la gerencia moderna inculque la importancia de hacer realidad la responsabilidad social, la participación de la empresa en la vida económica-productiva del país será mucho más significativa. Por otra parte se plantea, que las organizaciones socialmente responsables, están recurriendo a sus valores y experiencias del pasado y a su preocupación por el presente para establecer una nueva posición de responsabilidad para el futuro.

Una empresa no debe descuidar dentro de su responsabilidad social aspectos como:

- Ofrecer productos y servicios que respondan a necesidades de sus usuarios, contribuyendo al bienestar.

- Tener un comportamiento que vaya más allá del cumplimiento de los mínimos reglamentarios, optimizando en forma y contenido la aplicación de todo lo que le es exigible.

- La ética ha de impregnar todas las decisiones de directivos y personal con mando, y formar parte consustancial de la cultura de empresa.

- Las relaciones con los trabajadores han de ser prioritarias, asegurando unas condiciones de trabajo seguras y saludables. Ha de respetar con esmero el medio ambiente. 
- Ha de integrarse en la comunidad de la que forma parte, respondiendo con la sensibilidad adecuada y las acciones sociales oportunas a las necesidades planteadas, atendiéndolas de la mejor forma posible y estando en equilibrio sus intereses con los de la sociedad.

Diversos organismos internacionales como la Comisión Económica para América Latina y el Caribe (CEPAL), el Instituto Ethos de Responsabilidad Social, el European Bahái Business Forum, el Business in the Community, la International Organization for Standardization (ISO), desarrollan propuestas para orientar, fomentar, regular y promover la RSE. En este sentido, la ISO propone un documento denominado norma ISO 26000, en el cual establece una guía para apoyar a las empresas a demostrar de forma transparente su compromiso con los grupos de interés e incrementar la credibilidad de sus informes en materia de RSE. La ISO 26000 establece 7 materias fundamenta les en su guía para la operacionalización de la RSE: derechos humanos, prácticas laborales, gobernabilidad, medio ambiente, prácticas justas de operación, asuntos de consumidores, participación activa y desarrollo de la comunidad. La norma es aplicable a cualquier tipo de empresa, independientemente de su tamaño, y otorga una particular importancia al capital humano que labora en la empresa. (López Salazar, J.F., \& Manríqueza, 2017)

A decir de (Puterman, 2014) la ISO 26000 promueve el entendimiento común de la responsabilidad social ya que:

- Fomenta que las organizaciones realicen actividades que vayan más allá del cumplimento legal.

- Presenta en forma clara los antecedentes, tendencias y características de la responsabilidad social.

- Define los principios y prácticas relativos a la responsabilidad social.

- Presenta una dimensión estratégica de la responsabilidad social y el enlace entre la organización, la sociedad y las partes interesadas.

- Presenta las materias y asuntos fundamentales relacionados con la responsabilidad social a través de un enfoque holístico.

- Orienta sobre cómo integrar, implementar y promover un comportamiento socialmente responsable en toda la organización y a través de sus políticas y prácticas relacionadas con su esfera de influencia. 
- Ayuda a identificar a las partes interesadas y da razones para su involucramiento.

- Dibuja líneas sobre cómo determinar la pertinencia e importancia de las materias y asuntos fundamentales de la responsabilidad social.

- Ofrece líneas de cómo evaluar la esfera de influencia y sobre cómo ejercer influencia sobre ella.

- Promueve la comunicación de los compromisos y el desempeño relacionados con la responsabilidad social.

- Se complementa con otros instrumentos e iniciativas relacionadas con la responsabilidad social y, de hecho, así lo ha demostrado el Pacto Global y el GRI.

- Promueve el aumento de la toma de conciencia y creación de competencias en responsabilidad social.

- Orienta sobre cómo establecer el rumbo de la organización en materia de responsabilidad social.

- Orienta sobre cómo incorporar la responsabilidad social dentro de la gobernanza, los sistemas y procedimientos de una organización.

- Refuerza el rol de la comunicación y da ideas de cómo comunicar.

- Recomienda cómo mejorar la credibilidad en materia de responsabilidad social.

- Recomienda cómo mejorar el desempeño.

- Da orientaciones para las políticas de desarrollo; la eficiencia, la gobernabilidad y la rendición de cuentas.

- Es una herramienta para ayudar a las empresas, gobiernos, asociaciones, entidades y organizaciones no gubernamentales a incorporar la responsabilidad social como un principio de buena gestión.

- Contribuye al desarrollo de relaciones sólidas y de confianza mutua, y a los beneficios entre las organizaciones y sus grupos de interés.

- Es compatible con otros sistemas de gestión (ISO 9000, ISO 14000, OSHAS 18001 ySGE1).

- Brinda una oportunidad para que las organizaciones logren una ventaja competitiva y sustentable mediante la integración de la sostenibilidad y de la responsabilidad social en su visión y estrategia. 
Este trabajo tiene como objetivo, exponer teóricamente el tema de la responsabilidad social empresarial.

Para ello se muestra qué se entiende por responsabilidad social, se aborda el proceso histórico de la responsabilidad social, se explicitan los problemas actuales por acciones no responsables socialmente, los factores que afectan los sentimientos del grupo empresarial y los factores que se oponen a la productividad; se tratan los actos responsables de la empresa y las ventajas de la responsabilidad social de las empresas.

Por medio del análisis documental es que se realiza este trabajo, se localiza bibliografía relevante, se analiza y se sintetizan los objetivos de lo general a lo particular.

\section{Desarrollo}

La responsabilidad social, no es algo novedoso en el mundo empresarial, aunque en estos últimos años está adquiriendo una nueva dimensión por el especial interés con respecto al tema que involucra a trabajadores, clientes, proveedores y la sociedad en general.

Una empresa existe para producir mercancías o servicios que la sociedad desea y necesita (además de beneficios), y ello en un clima de estabilidad y confianza. El cumplimiento de este objetivo es su primera y principal responsabilidad. Si fracasa en esta misión, no puede esperarse que asuma otras.

La industrialización exige la voluntad de invertir hoy para el mañana y ésta faltará si el mañana es desconocido o repleto de riesgos. La estabilidad social proporciona el marco para la planificación y la inversión, con la esperanza de que los frutos de estos esfuerzos puedan conservarse. La empresa, sus propietarios y otros partícipes dependen de la sociedad donde operan en cuanto a su existencia y prosperidad, pero también tienen respecto a ella importantes obligaciones.

Con certeza, el motor que mueve a gestionar la Responsabilidad Social en empresas importantes no es puramente financiero, se alimenta de forma proactiva y voluntaria, más allá de los aspectos solidarios, en una mejora de la reputación y en una obtención de ventajas competitivas con una visión de negocio a largo plazo. Por tanto, en todo momento deben verificarse las elecciones para adoptar las estrategias más oportunas a tenor de los distintos criterios de los beneficios y la coyuntura socioeconómica del medio. 
La responsabilidad social empresarial vista desde un enfoque teórico

Según el Departamento de Estudios sobre Dirección del Trabajo (2005), la responsabilidad social empresarial (RSE), es una nueva forma de hacer empresa, que se vincula en su desarrollo reciente al proceso de globalización de la economía mundial, surge del convencimiento de que la necesidad de innovación empresarial para la competitividad y la sustentabilidad del negocio, debe ir más allá de lo puramente tecnológico para instalarse definitivamente e infinitivamente en el ámbito de la gestión y de la relación de la empresa con su entorno social y medio ambiental y, particularmente, en el espacio de lo laboral. (Departamento de Estudios sobre Dirección del Trabajo, 2005)

La Responsabilidad Social Empresarial (RSE), puede definirse como la contribución activa y voluntaria al mejoramiento social, económico y ambiental por parte de las empresas, generalmente con el objetivo de mejorar su situación competitiva y valorativa y su valor agregado. (Infante Rivas, 2015)

La responsabilidad social empresarial o corporativa va más allá del cumplimiento de las leyes y las normas, dando por supuesto su respeto y su estricto cumplimiento.

El cumplimiento de estas normativas básicas no se corresponde con la Responsabilidad Social, sino con las obligaciones que cualquier empresa debe cumplir simplemente por el hecho de realizar su actividad. Sería difícilmente comprensible que una empresa alegara actividades de Responsabilidad Social Empresarial si no ha cumplido o no cumple con la legislación de referencia para su actividad. (Pérez Urquía, 2014)

Los antecedentes de la RSE se remontan al siglo XIX en el marco del Cooperativismo y el Asociacionismo que buscaban conciliar eficacia empresarial con principios sociales de democracia, autoayuda, apoyo a la comunidad y justicia distributiva. Sus máximos exponentes en la actualidad son las empresas de Economía Social, por definición Empresas Socialmente Responsables. (Rivarola Ganoza, 2013)

Para la Organización Internacional del Trabajo (OIT) la responsabilidad social de la empresa es el conjunto de acciones que toman en consideración las empresas para que sus actividades tengan repercusiones positivas sobre la sociedad y que afirman los principios y valores por los que se rigen, tanto en sus propios métodos y procesos internos como en su relación con los demás actores. La RSE es una iniciativa de carácter voluntario. Forma parte integrante de la gestión de la empresa. Es 
sistemática, no ocasional. Guarda relación con el desarrollo sostenible. No reemplaza el papel que desempeñan las autoridades públicas, ni a la negociación colectiva, ni a las relaciones de trabajo (OIT, $\mathrm{s} / \mathrm{f})$.

La responsabilidad social de la empresa es una combinación de aspectos legales, éticos, morales y ambientales, y es una decisión voluntaria, no impuesta, aunque exista cierta normatividad frente al tema. (Lugo Marín, 2015)

El concepto responsabilidad social empresarial o corporativa (RSE), tiene diversas acepciones, dependiendo de quién lo utilice. La conceptualización más holística y progresista hace referencia a que una empresa es socialmente responsable, cuando en su proceso de toma de decisiones valora el impacto de sus acciones en las comunidades, en los trabajadores y en el medio ambiente e incorpora efectivamente sus intereses en sus procesos y resultados. Asimismo, ejerce un especial respeto por las regulaciones y leyes internas del país donde opera y que la sociedad ha definido como válidas y legítimas, pero también respeta los acuerdos y tratados internacionales sobre fiscalidad, prevención de la corrupción, respeto a los derechos humanos y derechos laborales, protección del medio ambiente y busca garantizar que cumplan estas regulaciones y principios, los subcontratistas, socios comerciales, proveedores y cualquier otro con quien realice negocios. (Departamento de Estudios sobre Dirección del Trabajo, 2005)

Orientar los esfuerzos de la empresa solamente a producir resultados basados en las teorías de producir y vender más con el mínimo costo sin importar el impacto social, es a lo largo del tiempo, el peor negocio del sector productivo que busca la rentabilidad. La estrategia actual y sus esperados beneficios, con dicha posición, puede ser mañana el motivo de su fracaso. La responsabilidad social de la empresa abarca aspectos internos y externos, los que se han sido objeto de tratado por expertos en el tema, los primeros orientados a los colaboradores o el equipo de trabajo, sus asociados y accionistas, y los segundos, los externos a clientes, proveedores, familia de los trabajadores, la vecindad y el entorno social, entre estos el medio ambiente. (cap, 2017)

Los problemas por la inadecuada responsabilidad social no es causa generada por una sola fuente, es decir, por la empresa, todos los estamentos de un estado son igualmente responsables de sus males: las políticas blandas, imprecisas e irresponsables de los gobiernos; gobernantes permisibles y transigentes; los sistemas de corrupción; la poca preocupación e importancia concedida en el tema por 
La responsabilidad social empresarial vista desde un enfoque teórico

parte de entidades educativas; la ignorancia misma o falta de conocimiento sobre el tema por parte de empresarios y actores del sector productivo y comercial; el bajo escrúpulo de muchos empresarios y personas que conociendo el tema y sus consecuencias, actúan de forma irresponsable. (Guibert, 2013)

Es decir, todos los estamentos que hacen parte del estado, gobierno y sus políticas, colegios y universidades, empresas, empleados, padres de familia, medios informativos y demás que inciden en patrones de comportamiento y en la cultura, son igualmente responsables en los daños ocasionados, bien por acciones directas o por omisión.

El tema ambiental es altamente alarmante, y ha sido generado por el bajo interés en el asunto, y por la actuación no responsable del sistema productivo mundial. Ellos son: (Velásquez, 2011)

- El creciente calentamiento global en los últimos 160 años y sus efectos en el planeta: El deshielo y derretimiento de los casquetes polares que para el 2.100 aproximadamente, dicen los expertos, serán la causa de que los mares suban hasta metro y medio, desapareciendo naciones enteras y cambiando el mapa mundial.

- El aumento de sustancias productoras de la lluvia ácida y por ende el deterioro de bosques y extinción de especies animales y vegetales vitales para la armonía del ecosistema.

- El aumento de la contaminación de aguas y el envenenamiento de especies vegetales y animales, la contaminación para el riego de cultivos productores de alimentos, la proliferación de enfermedades por ésta vía y sus demás consecuencias que generan problemas de salud pública en un alto estado de alerta mundial.

- La sequedad de la tierra y la infertilidad de la misma, la deforestación y la reducción de vegetación como única fuente de producción de oxígeno y recuperación del aire.

- La extinción de especies animales y vegetales, con sus consecuencias negativas por el desequilibrio en la cadena alimenticia.

- El incremento del efecto invernadero y la alteración de los habitas.

- La reducción de la capa de ozono y los peligros por la exposición directa a los rayos del sol tanto para ser humano como para todas las especies vivas.

- Y otros tantos asuntos que en estos momentos son objeto de reglamentaciones y de concientización, como el incremento de basuras y la disposición final de las mismas, la 
La responsabilidad social empresarial vista desde un enfoque teórico

producción de alimentos cargados con contaminantes cancerígenos y posiblemente hasta agentes que estimulan mutaciones en los seres vivos.

Pero estos no son los únicos problemas que amenazan a la humanidad y a la empresa, hay otros que día a día crecen y que se convierten en la fatalidad para el futuro.

En la actualidad, reina una situación de desconcierto y desorden que hace que la práctica empresarial sea cada vez más compleja por no llamarle difícil, afectando el ejercicio financiero, no solamente por el reforzamiento de sistemas de seguridad personal, industrial o de los bienes, también por el crecimiento de ausentismos, por los daños causados en propiedad ajena, por las devoluciones, por los reprocesos, la pérdida de clientes, la inmoralidad de algunos funcionarios del mismo estado y más aspectos que hacen parte de lo que llaman "lo normal en un ejercicio empresarial", y que pueden ser medibles en los balances financieros de cada empresa. (Moya Arana, 2014)

El panorama actual para la empresa no es nada alentador, de nada favorece sus actividades, atenta contra la estabilidad financiera, afecta el bienestar interno y externo y por ende la productividad repercutiendo en la competitividad. Entre los hechos negativos se encuentran: (Moya Arana, 2014)

$\checkmark$ La creciente violencia y los conflictos sociales que amenazan la tranquilidad ciudadana, al sujeto y sus bienes y los de las empresas, implicando más inversión en seguridad, temor en invertir, y en caso de atentados o daños, la perdida y la consecuente reposición de los bienes.

$\checkmark$ La falta de identidad, el poco sentido de pertenencia por la misma región, país y empresa, y el compromiso ausente frente a planes de mejoramiento.

$\checkmark$ El estrés y los estados depresivos que generan ausentismos, deserciones, conflictos, tensiones $\mathrm{y}$ una serie de malestares que atentan con el ambiente organizacional.

$\checkmark$ Problemas emocionales, neurosis, paranoias sociales y otros estados que contribuyen a la accidentalidad, que desmotivan e influyen en la poca concentración y en bajos resultados.

Los anteriores entre muchos de los que podemos enumerar, pero igualmente hay otros males que estropean lo personal y desestabiliza a los grupos familiares, con efectos en los grados de atención, la disposición, el aporte y los resultados en la empresa y en general a la sociedad. Entre algunos: (Moya Arana, 2014) 
$\checkmark$ La creciente violencia y los conflictos sociales que amenazan la tranquilidad ciudadana, al sujeto y sus bienes y los de las empresas, implicando más inversión en seguridad, temor en invertir, El aumento de la industria del secuestro, las violaciones, el atraco y otros actos violentos que generan temores, zozobras y crean un clima de tensión y miedo.

$\checkmark$ El aumento de robos, de hurtos, de desfalcos y otras prácticas deshonestas que reducen la motivación hacia la inversión, implican más costos en protección y que por el mismo estado de prevención, aminora competencias y estados de ánimo, pues se crece la desconfianza, los estados de recelo y las duda.

Junto a otros asuntos lo anterior hace parte del panorama en que se desenvuelven las personas y en el que está inmerso la empresa. Un escenario muy criticado pero que pereciera inmodificable, por la fuerza que con el tiempo ha adquirido, convirtiéndose en lo normal de la vida cotidiana.

Es importante aclarar que la productividad de las personas se ve seriamente afectada cuando se encuentra estropeada emocionalmente, y por ende los resultados de la empresa, así que, si el ambiente en que se encuentra la sociedad, y por ende la empresa, es sano y favorable, la productividad y la competitividad será mayor, pero de no ser así, se manifestará en sus resultados, de producción, de mercadeo y por ende financieros.

Entre esos factores que afectan sentimientos y la tranquilidad del grupo laboral, y que aunque aparentemente no son de responsabilidad directa de la empresa, pero sí repercuten en los sus resultados, se encuentran: (González S., 2007)

- Las bajas relaciones inadecuadas de pareja y el crecimiento de conflictos familiares.

- Incremento en los índices de padecimientos de anorexia y bulimia en adolescentes.

- Prostitución progresiva, inclusive infantil.

- Altas tendencias de homosexualidad.

- Crecimiento de pandillas juveniles.

- Practicas satánicas.

- Alta infidelidad.

- El crecimiento de los índices de suicidio.

- Otras. 
La responsabilidad social empresarial vista desde un enfoque teórico

Esto produce decaimiento emocional y se convierte en razón para el ausentismo, la deserción laboral, la accidentalidad en el ejercicio de las funciones de trabajo, la apatía, el crecimiento de conflictos, la baja motivación por lo que se hace y demás manifestaciones que merman la capacidad de concentración y la disposición hacia la productividad, además llegan a afectar en aspectos como la pérdida total de interés por lo que se hace y por la misma vida, se acaba la ilusión.

A esto se le puede sumar otra cantidad de actitudes, comportamientos, hábitos, costumbres y formas de pensar, que se oponen al buen desempeño del grupo de trabajadores y por ende a la productividad de una compañía, entre ellas: (González S., 2007)

- El negativismo o pesimismo. Reduce la motivación por los propósitos organizacionales.

- La apatía al estudio, al mejoramiento continuo y la tendencia al facilismo.

- La baja disposición al ahorro y por ende los consecuentes problemas que genera el mal manejo de los ingresos, con sus posibles consecuencias negativas para obtener lo que desea. Un problema financiero es potencialmente el cultivo de actos indelicados.

- La baja disposición por aceptar una crítica constructiva, lo que conlleva a la generación de posiciones negativas para con quien la hace.

- Bajo sentido de identidad y de pertenencia, desencadenando conductas poco participativas, se hace las cosas tan solo por cumplir sin dar un algo más a favor de los propósitos de la empresa.

- El conformismo, la marrullería, que reduce la capacidad de aporte así como la de auto crecimiento o mejoramiento personal.

- El facilismo con un bajo sentido de auto mejoramiento y de auto aprendizaje, por ende poca capacidad de aporte para la empresa.

- Altas tendencias a tomar frívolamente las cosas, con mucha inclinación a lo festivo, concediéndole mayor prioridad a ello que al trabajo mismo.

Definitivamente enumerar la cantidad de aspectos que hacen parte hoy de un gran grueso de nuestra población laboral es una tarea casi interminable, y todo lo anterior repercute en la perdida de la esperanza en las personas, la baja ilusión, la poca disposición por hacer bien las cosas, merma su capacidad productiva y afecta como lo he indicado la productividad, la que se refleja en los estados financieros de las empresas. 
Las empresas son partícipes de la responsabilidad en algunos de los efectos mencionados, o en todos, por sus políticas o la carencia de las mismas, por sus sistemas de dirección y por una serie de costumbres y vicios empresariales que le alejan de una actuación responsable que mida la consecuencia de sus actos.

La falta de ética y moral, es sin lugar a duda, el principal elemento del problema, la búsqueda de las utilidades y el afán de generar resultados a toda costa sin medir consecuencias, no permite que la empresa (los empresarios y directivos), presten la atención que merece el tema de la responsabilidad social.

La inmoralidad ciudadana, la falta de civismo, el bajo escrúpulo de las personas, la falta de lealtad, la marrullería, la trampa, la poca seriedad, el bajo sentido de pertenencia, la mediocridad misma, las inadecuadas relaciones humanas, así como esos aspectos, son muchos los asuntos de comportamiento que afectan el desarrollo empresarial y por ende su eficiencia, y podré demostrar que la empresa tiene una alta responsabilidad en ellos.

El CEMEFI, en sus revisiones, incluye cuatro ámbitos con los que certifica a las empresas y que debe cumplir toda empresa socialmente responsable (Porto \& Castromán, 2006):

1. Contribuir a la calidad de vida dentro de la empresa. Esto incluye la generación de empleos, el pago de salarios justos y oportunos, constante capacitación, comunicación eficaz, trato digno, el trabajo en equipo, etc.

2. Cuidado y preservación del medio ambiente. Es necesario que las empresas cuiden el entorno que les rodea y fomenten en los trabajadores y sus familias el ánimo para preservarlo. También se espera que exijan a sus proveedores, trabajadores, autoridades y gobierno que lo hagan porque no es suficiente cumplir con las normas ambientales sino que se requiere fomentar los valores para que esto sea voluntario y permanente, para lo que es posible y deseable contar con la ayuda de organismos especializados en cuestiones ambientales.

3. Desempeñarse con un código de ética. El trato ético permitirá a las empresas mantener relaciones de calidad con proveedores y clientes, resultando que más de ellos buscarán hacer negocio con esas empresas generando mayores volúmenes de venta y más ingresos como consecuencia. 
4. Vincularse con la comunidad a partir de la misión del negocio, pero también de los bienes y servicios producidos. Esto incluye la colaboración activa y voluntaria con la sociedad mediante la participación en programas de apoyo como la donación de recursos a diversas fundaciones de ayuda humanitaria, planes de becas para niños y jóvenes, así como asistencia técnica para obras de beneficencia promovidas por organismos sin fines de lucro, entre otros.

Asimismo para el CEMEFI (Porto \& Castromán, 2006) este debe ser el "decálogo" de toda empresa que desee ser socialmente responsable:

1. Promover e impulsar en su interior una cultura de RSE que busque las metas y el éxito del negocio, contribuyendo al mismo tiempo al bienestar de la sociedad.

2. Identificar las necesidades sociales del entorno en que opera y colaborar en su solución, impulsando el desarrollo y mejoramiento de la calidad de vida.

3. Hacer públicos los valores de su empresa y desempeñarse con base en un código de ética.

4. Vivir al interior y al exterior de esquemas de liderazgo participativo, solidaridad, servicio y respeto a la dignidad humana.

5. Promover el desarrollo humano y profesional de toda su comunidad (empleados, familiares, accionistas y proveedores).

6. Identificar y apoyar causas sociales como parte de su estrategia de acción empresarial.

7. Respetar el entorno ecológico en todos y cada uno de los procesos de operación y comercialización, además de contribuir a la preservación del medio ambiente.

8. Invertir tiempo, talento y recursos en el desarrollo de las comunidades en las que opera.

9. Participar, mediante alianzas intersectoriales con otras empresas, en organizaciones de la sociedad civil (OSC) y/o con el gobierno, en la atención de las causas sociales que ha elegido.

10. Tomar en cuenta e involucrar a su personal, accionistas y proveedores en sus programas de inversión y desarrollo social.

11. La Responsabilidad Social Empresarial (RSE) es un elemento útil y eficaz para mejorar notablemente el modelo productivo de una empresa, porque une los criterios de eficiencia, sostenibilidad y prosperidad con al añadido valor de la diversidad, igualdad de oportunidades, respeto, tolerancia, etc.

12. La RSE por si constituye una gran oportunidad de mejorar los beneficios y bondades de las empresas, ya sean grandes o pequeñas. Varios estudios demuestran que las empresas que 
avanzan y progresan en las políticas de RSE obtienen más productividad lo que repercute directamente en los resultados.

Entre los beneficios para la empresa, se puede destacar: (EOI, 2014)

$\checkmark$ Mejor capacidad de contratación y permanencia de los empleados. Las personas admiran a las empresas que valoran a sus empleados, que les dan soporte y ayuda continua, aun en momentos difíciles. Además que los empleados se sienten más motivados y a gusto con el comportamiento de dicha empresa como tal. Esto estimula a que los empleados permanezcan en la empresa y valore su empleo.

$\checkmark$ Mayor lealtad del consumidor: Los consumidores admiran aquellas empresas que valoran a sus empleados, desarrollan proyectos sociales, se preocupan por el medio ambiente e invierten esfuerzos contra la corrupción. Por ello, la responsabilidad social es factor que garantiza la fidelidad del consumidor.

$\checkmark$ Valoración de la imagen y de la marca. Las prácticas de responsabilidad social agregan valor a los negocios de la empresa, la cual puede valerse de ese potencial diferenciador para sus estrategias de negocios.

$\checkmark$ Sostenibilidad del negocio a largo plazo. La perennidad de la empresa constituye también motivo de preocupación para los inversionistas. Las prácticas socialmente responsables disminuyen las contingencias de la empresa y permiten su control, reduciendo el riesgo del negocio como un todo.

En este mismo sentido se plantea (Nakamura, 2015)

1. Mejorar la imagen corporativa y la reputación: Actualmente estamos viviendo lo que los expertos catalogan como "la economía de la reputación". En unos mercados en los que todo se copia, los intangibles - marca y reputación - han pasado a convertirse en variables fundamentales para diferenciarse. Una reputación cuya construcción se ha transformado también en los últimos tiempos. La empresa sigue siendo el actor principal, pero ya no el único. Hoy las aportaciones e interacciones de clientes, trabajadores, proveedores y otros grupos de interés, juegan un papel esencial. 
2. Previene riesgos. La RSC es también un instrumento muy eficaz para reducir los riesgos potenciales asociados a las actividades que desarrollan las empresas.

La creación de códigos éticos y de conducta, el establecimiento de estándares responsables en la selección de proveedores o la incorporación de consideraciones medioambientales o de Derechos Humanos a lo largo de la cadena de valor, reduce significativamente la probabilidad de que las compañías incurran en "malas praxis". Al reforzar el control de las operaciones que realiza una empresa, esta logra minimizar los conflictos con las comunidades en las que opera; disminuir quejas y procesos judiciales, mejorar las relaciones con clientes, empleados, sindicatos, administraciones públicas y otros grupos de interés y reducir drásticamente la posibilidad de sufrir riesgos como publicidad negativa o boicots a sus productos o servicios.

3. Captación y retención de talento. Cuando valoran un empleo, el factor económico pierde peso frente a variables como la adecuación del mismo a su proyecto de vida. Buscan adherirse a causas, prefieren empresas comprometidas con la sociedad y el medioambiente y valoran los entornos que promueven la multiculturalidad y la diversidad. En este escenario, la RSC ayuda a diseñar y ofrecer propuestas novedosas y atractivas para este nuevo perfil de trabajadores el s XXI.

4. Mejora el clima laboral y de la productividad. Junto a la captación y retención de talento, una buena estrategia de RSC tiene efectos muy positivos en el grado de satisfacción y compromiso de los trabajadores con la compañía. La implementación de programas de voluntariado corporativo ofrece infinidad de ventajas. Mejora la motivación, las habilidades y la autoestima de los trabajadores; fomenta el orgullo formar parte de la empresa y refuerza la implicación de los empleados y su compromiso con la misma.

Una adecuada gestión de la diversidad incide positivamente en términos de productividad y competitividad, lo que incrementa la probabilidad de encontrar soluciones más innovadoras y robustas.

5. Fomenta la innovación. La RSC incentiva el desarrollo de productos y servicios innovadores. El diálogo con los diferentes grupos de interés, unido a la búsqueda soluciones vinculadas a 
La responsabilidad social empresarial vista desde un enfoque teórico

objetivos de sostenibilidad e impacto social, potencian el desarrollo de productos novedosos que permiten el acceso a nuevos segmentos de mercado.

6. Mejora la eficiencia energética. Los objetivos medioambientales que asumen las empresas en el marco de sus acciones de RSC suelen incluir medidas de eficiencia energética, reciclado y otras iniciativas de disminución de consumo de recursos naturales. Con ello, además de minimizar sus impactos negativos sobre el medio ambiente, obtienen un significativo ahorro en términos económicos.

7. Trae ventajas para atraer financiamientos. Un comportamiento responsable implica la implementación de prácticas de buen gobierno, éticas y transparentes que inciden favorablemente en su credibilidad en los mercados y mejoran su acceso a financiamiento. Además de ventajas fiscales, las empresas responsables reducen el riesgo de contingencias, ofreciendo con ello un valor añadido que incrementa el valor de sus acciones y refuerza la confianza de inversores reales y potenciales.

8. Aumenta la fidelización de consumidores y clientes. Los consumidores admiran aquellas empresas que valoran a sus empleados, desarrollan proyectos sociales, se preocupan por el medio ambiente e invierten esfuerzos contra la corrupción. Por ello, la responsabilidad social es un factor esencial para garantizar la fidelidad del consumidor. Una tendencia al alza, teniendo en cuenta los nuevos hábitos de consumo de las generaciones venideras.

9. Facilita la obtención de premios y reconocimientos. Son muchos los premios y reconocimientos que están surgiendo en materia de responsabilidad y sostenibilidad. Los premios además de reconocer de manera pública los esfuerzos de las empresas en este ámbito, ayudan positivamente a difundir las iniciativas de las compañías a través de foros no empresariales. Asimismo, mejoran la relación con el entorno, logran una mayor credibilidad frente a nuevos consumidores y usuarios e incrementan la influencia de la empresa en su sector.

10. Sitúa a la empresa en la preferencia para la contratación pública y las licitaciones. Son muchos los gobiernos o entidades que están incorporando consideraciones de sostenibilidad y 
La responsabilidad social empresarial vista desde un enfoque teórico

responsabilidad social como criterios de valoración y mejora para seleccionar a los adjudicatarios en los procesos de contratación pública.

\section{Conclusiones}

Es evidente las ventajas que ofrecer ser una empresa responsable, sin embargo éstas no se comportan de modo responsable por el mero hecho de decir que aceptan los valores enunciados en este trabajo y obtener con ello una ventaja competitiva sobre las otras empresas. No basta el sólo compromiso, este debe ir sujeto a la implementación de las acciones para una empresa verdaderamente responsable. Las empresas deben tomar en cuenta las consecuencias éticas, sociales y ecológicas de sus actividades, productos y servicios.

Para lograr respetar el medio ambiente, ofrecer calidad laboral a los empleados, conducirse con ética en las relaciones con sus grupos de interés, respetar al consumidor y participar activamente con la comunidad es importante la conciencia de todos los empleados, un liderazgo empresarial y una infraestructura adecuada.

\section{Referencias Bibliográficas}

CAP. (2017). RSE como herramienta de publicidad. Recuperado el 12 de abril de 2017, de http://revistacap.com/publicidad1/181-rse-como-herramienta-de-publicidad

Departamento de Estudios sobre Dirección del Trabajo. (2005). Responsabilidad social empresarial, alcances y potencialidades en materia laboral. Recuperado el 20 de abril de 2017, de http://www.dt.gob.cl/1601/articles-88984_recurso_1.pdf

EOI. (2014). Los Beneficios de la Responsabilidad Social Empresarial (RSE) para las empresas. Recuperado el 02 de febrero de 2017, de http://www.eoi.es/blogs/embacon/2014/02/03/losbeneficios-de-la-responsabilidad-social-empresarial-rse-para-las-empresas/

García, O. (2014). Liderazgo Gerencial. Recuperado el 04 de febrero de 2017, de https://ogarcia3086.wordpress.com/2014/08/13/liderazgo-gerencial/

González S., T. (2007). La responsabilidad social de la empresa, un buen negocio. Recuperado el 25 de febrero de 2017, de http://www.sld.cu/galerias/pdf/sitios/infodir/responsabilidad_social.pdf 
La responsabilidad social empresarial vista desde un enfoque teórico

Guibert, M. (2013). El rol del contador público y su responsabilidad ética ante la sociedad. Perú: Tesis para optar el grado académico de Doctor en Contabilidad y Finanzas.

Infante Rivas, H. (2015). Responsabilidad social corporativa o empresarial. Recuperado el 05 de febrero de 2017, de https://www.gestiopolis.com/responsabilidad-social-corporativa-o-empresarial/

López Salazar, A., J.F., O. H., \& Manríqueza, M. (2017). La responsabilidad social empresarial desde la percepción del capital humano. Estudio de un caso. Revista de Contabilidad.

Lugo Marín, J. (2015). La Gerencia de la Calidad en la Economía Digital.

Moya Arana, I. (2014). Responsabilidad social.

Nakamura, P. (10 de febrero de 2015). La importancia de la RSC: 10 ventajas claves para la empresa. Recuperado el 18 de febrero de 2017, de https://patricianakamura.com/2015/03/15/la-importancia-dela-rsc-rse-10-ventajas-claves-para-la-empresa/

OIT. (s/f). La OIT y la responsabilidad social. Obtenido de http://www.ilo.org/wcmsp5/groups/public/---ed_emp/---emp_ent/---

multi/documents/publication/wcms_142694.pdf

Pèrez Urquìa, R. (2014). Gestión de recursos laborales, formativos y análisis de puestos de trabajo. Juvenile Nonfiction.

Porto, N., \& Castromán, j. (2006). Responsabilidad social: un análisis de la situación actual en México y España. Contaduría y Administración, Núm. 220.

Puterman, P. (20 de noviembre de 2014). ¿Se agotó la RSE? Diario Responsable. Obtenido de Diario responsable.

Rivarola Ganoza, I. (2013). Historia de la responsabilidad social y el desarrollo sostenible. Recuperado el 18 de febrero de 2017, de https://www.gestiopolis.com/historia-de-la-responsabilidadsocial-y-el-desarrollo-sostenible/

Velásquez, S. (2011). Responsabilidad Social Corporativa. UNAH. 